\title{
Adaptive Dynamic Surface Control of a Flexible Robot Based on the K-State Observer
}

\author{
Lei Yang ${ }^{1}$, Chuansheng Tang ${ }^{1, *}$, Jie Yang ${ }^{2,3,4}$, Fuyang Liu' ${ }^{5}$ and Tao $\mathrm{Li}^{6}$ \\ ${ }^{1}$ Nanyang Institute of Technology, Nanyang Henan 473000 China \\ ${ }^{2}$ General Graduate School of Dongseo University, Busan 47011, Korea \\ ${ }^{3}$ Henan Institute of Technology, Xinxiang Henan 453000, China \\ ${ }^{4}$ Henan Key Equipment Engineering Research Center for New Energy Power Generation, Xinxiang Henan 453000, China \\ ${ }^{5}$ Xiangyang public inspection and Testing Center, Xiangyang Hubei 441100, China \\ ${ }^{6}$ Department of Informatics, University of Zurich, Zurich 8050, Switzerland
}

Received 3 September 2020; Accepted 25 November 2020

\begin{abstract}
The flexible manipulator is a complex electromechanical nonlinear system with a rigid flexible coupling, and its control performance is affected by many factors, such as inaccurate modelling and measurement, load variation, and uncertainty of external disturbance. In view of improving the effect of time-varying model parameters and external disturbances on the performance of the control system, an adaptive dynamic surface control method of the flexible manipulator based on K-state observer was proposed in this study. On the basis of the analysed characteristics of the flexible manipulator system model, a K-state observer had been constructed when the system state was not completely measurable. Then, an adaptive dynamic surface control method was designed by taking the position and speed of the flexible manipulator as the control objective. Finally, the effectiveness of the control method was verified by simulation. Results show that, when no model parameter uncertainty exists in the system, the dynamic surface control has a higher tracking accuracy than the inversion control, the maximum tracking error of angular velocity is reduced by 1.3 times, and the steady-state control input is reduced by 550 times. When the system has model parameters but only the joint angle position can be measured, the conventional inversion control and dynamic surface control cannot achieve system control. The adaptive dynamic surface control can achieve high-precision system tracking at $2.5 \mathrm{~s}$, and the state and parameter estimation of the system can achieve online observation at $4 \mathrm{~s}$ and $2.5 \mathrm{~s}$, respectively; and the adaptive dynamic surface control in the steady-state control input can reach $1 / 18$. The proposed method can provide a reference for a flexible robot to achieve high-precision tracking in a complex assembly environment.
\end{abstract}

Keywords: Flexible joint robot, Dynamic surface control, K-state observer, Inverse control, Adaptive estimation

\section{Introduction}

The development of robot systems towards the direction of high-speed, heavy-load, high-precision, and lightweight apparatuses will inevitably increase the elastic deformation of components, which conflicts with the stability and accuracy of robotic motion-a concern that highlights the impact of elastic deformation of various components on the performance of robots. In addition, the manipulator of largescale machinery, such as aviation equipment, vehicle robots, and buildings, must be connected by some small cross parts relative to the lengths of these parts to ensure flexibility, but such a configuration leads to mechanical vibrations that may hinder the easy control at the end position. The traditional study methods of rigid robots are obviously unsuitable for the study of this kind of robot [1]. Consequently, a new kind of flexible robot has become a hot spot of robot research.

Flexible robots offer high-speed, low-energy consumption, and low-impact advantages, especially in the aerospace and manufacturing fields that use light and longspan space manipulators [2], deployable antennas [3] and solar arrays [4], high-speed and high-precision assembly robots [5], or high-speed laser cutting robots [6], etc. However, the flexible robot is a highly complex dynamic

*E-mail address: tcs111@163.com

ISSN: 1791-2377 @ 2020 School of Science, IHU. All rights reserved. doi:10.25103/jestr.136.23 system with highly nonlinear, multi-time-varying, and strong coupling characteristics. Numerous uncertain factors, such as accuracy of modeling and measurement, uncertainty of parameters, incompletely measurable state, external disturbance, and friction between joints, also need to be considered. These uncertain factors are the key elements in realizing the stability control of flexible robots, especially for robot systems under working conditions requiring highspeed, high-precision, high-performance, and complex configuration. Thus, the high-performance control problem particularly needs to be studied along with the parameter uncertainty and incompletely measurable system state.

Flexible robots are developing towards apparatuses with multiple degrees of freedom (DOF), polarization, and direct drive mechanisms. Asymmetric multi-DOF flexible hand grasping based on a flexible mechanism can realize the mechanized and stable picking of fruits and vegetables with different shapes and structures [7]. A high-precision microdetection robot can precisely observe an oil-immersed transformer moving along a specific depth direction [8]. A heavy-duty friction stir welding robot can realize dexterous welding under heavy-duty conditions in a large working space and realize high-strength and high-precision welding operation for large-scale complex curved parts [9]. Through a closed-loop control, a flexible robot can complete its bolt tightening task during the high-precision assembly of an engine [10]. However, the actual working environment of 
robots is complex and changeable, and the parameters of robot models are uncertain when the environment changes. Consequently, measuring completely a system state is rarely realized. Thus, the corresponding control methods must be studied to be able to achieve high-precision control.

An equivalent system state model can be established by model state transformation according to the structural characteristics of the flexible robot. Here, a K-state observer is constructed, and a robust control method is designed on the basis of adaptive theory and dynamic surface control theory. The purpose of this method is to solve the control problem of the system under incomplete measurability and time-varying parameter conditions and subsequently provide a scheme for the high-precision tracking of the flexible robot.

\section{State of the art}

The development of applications for flexible robots has helped to flourish the study of the tracking control of these robot types. Most industrial objects can be operated using the proportional, integral, and differential (PID) control, whose structure is simple and easy to realize and thus is also the most commonly used control in industrial robots. To achieve the high-speed response and high-precision control of a flexible robot, $\mathrm{Fu}$ et al. [11] proposed a simple PID position tracking control method. The accuracy of the method was high when the system model could be determined and the load was constant, but the position and speed information of the robot joint and motor could not control parts of the state information location, such as motor position and speed information. By combining fuzzy control with PID control, Sarkhel et al. [12] designed a fuzzy gain PD control method to improve to a certain extent the dynamic performance of a system. However, the structure of the designed controller was complex, and its fuzzy rules were difficult to determine. To improve adaptability under different loads, Santanu et al. [13] proposed a new nonlinear self-tuning PID control method to attenuate unmodeled highorder dynamics by using a new non-linear autoregressive moving average with an external input model. The NSPIDC method helped to ensure stability with the change in payload. The parameters of the flexible manipulator were identified online by a recursive least-square algorithm using the minimum variance control (MVC) updating control parameters in real time. The MVC method needed to acquire information about the system state before it could be completely measurable. Malzahn et al. [14] analyzed the role of vibration damping on the joint position, velocity, and motor current regulation in the feedback control of a flexible robot and determined that control accuracy was highly dependent on the parameters of the system. Endo et al. [15] studied the stability of two single-link flexible arms for grasping and controlling the direction of objects. The flexible arm was modeled from the Euler Bernoulli model. The robustness of the closed-loop system to the disturbances distributed on the arm and boundary was discussed using the frequency domain method. Their proposed method also needed all of the information of the system, from state to control. To realize the contact force control of a flexible robot, Endo et al. [16] also proposed a simple boundary controller and used the frequency domain method to determine the exponential stability of a closed-loop system. Their method was not robust and unable to meet the requirements of a complex environment. Luc et al. [17] designed a robust adaptive control method based on the cascaded structure, and they used the full-state feedback controller and integral term as the internal control loop and then calculated torque as the external control loop. The robot had no chattering under zero-motor speed owing to the adaptive friction compensation, and the adaptive technology ensured the trajectory tracking of the robot. However, the premise of their work was that the robot model structure was completely known and linear with unknown parameters, and the information about joint speed was available. The proposed method was often used in combination with the sliding mode control, disturbance observer, and intelligent control. Palleschi et al. [18] applied optimal theory to the path planning of a flexible robot and transformed its optimal control problem into a convex optimization problem, which effectively realized the optimal control of the robot. This method was sensitive to model parameters but needed to be improved in complex environments. Aiming at the joint tracking control of a cable-driven flexible robot with a permanent magnet variable stiffness device, Zhang et al. [19] designed a decoupling control method of stiffness and position for the trajectory control. This method attained high tracking performance for the deterministic model, but the performance decreased when the system included uncertain parameters. Aiming at solving the dynamic rigid equation problem of a flexible joint robot and by combining the idea of the quantized state system and trapezoidal integral methods in implicit algorithm, Li et al. [20] put forward a step correction optimization algorithm based on a quantized state system, which effectively improved the accuracy and efficiency of dynamic solution, but its robustness was poor. Zhu et al. [21] considered the characteristics of the motor, and the singular perturbation theory was decomposed into fast and slow variable subsystems. For a fast-varying subsystem, speed difference feedback control was adopted. For a slow-varying subsystem, a full-order sliding mode control based on the radial basis function (RBF) neural network was proposed. The RBF neural network was employed to approach the unknown nonlinear term of the system. The full-order sliding mode offered the advantages of simple structure and strong robustness, and it could also overcome the chattering problem. However, not all of the state information could be determined in the system design. In view of adapting the changes in blade curvature in the grinding process, active and passive grinding force controls needed to be realized. Wang et al. [22] proposed a novel non-singular fast-terminal sliding surface and multi-power reaching rate and designed a control law with disturbance compensation based on the new sliding mode surface and multi-power approach rate. The method could realize fast dynamic response and anti-interference, but it could not eliminate the chattering caused by the sliding mode control. Xue et al. [23] combined the fuzzy control with the backstepping control and proposed a fuzzy back-stepping adaptive position control method for flexible robots. The rule of fuzzy control has no uniform form thus far, and it entails difficulties in application. To solve the trajectory tracking control problem of flexible joint robots under the condition of limited joint driving torque output, Liu et al. [24] proposed a back-stepping control method based on singular perturbation theory. The back-stepping control needed differential derivation in each step, which could cause knowledge explosion and other problems. Ouyang et al. [25] combined back-stepping and reinforcement learning of a robot with hand-grasping control, but this method could not eliminate knowledge explosion and other problems caused by the back-stepping control. For an exoskeleton 
flexible robot with parameter uncertainty and external disturbance, Brahmi et al. [26] designed a tracking control method for the robot with time-delay estimation and backstepping control method, and the method needed to know the velocity of the robot joint and the position and speed of the motor. Ultimately, the back-stepping control would lead to differential explosion, and the control input entailed large chattering, suggesting difficulties in meeting the requirements of high-precision tracking.

Most of the aforementioned studies have focused on the influence of a system model's parameter uncertainty; by contrast, few studies have aimed to determine the condition in which system states are not completely measurable. In the current study, based on the characteristics of the flexible robot model, the dynamic model of the system was reconstructed, the state of the system was observed by the $K$ state observer. The robust control method with high performance was designed to provide the basis for the application of a flexible robot in a complex and changeable environment.

The remainder of this study is organized as follows. Section 3 builds the dynamic model and the equivalent transformation model of the flexible robot, and the robust adaptive dynamic surface control method based on the Kobserver is designed to analyze the stability of the system. Section 4 verifies the effectiveness of the proposed control method based on the comparison with the traditional control method. Section 5 summarizes the conclusions.

\section{Methodology}

\subsection{System description}

A typical flexible joint robot is shown in Figs. 1 and 2. This kind of robot simplifies the connection between the drive motor and the joint into a spring, and the rotation angle of the motor has a certain deviation from that of the joint. The complex dynamic system model can be represented as follows:

$$
\left\{\begin{array}{l}
I \ddot{q}_{1}+M g l \sin q_{1}+K\left(q_{1}-q_{2}\right)=0 \\
J \ddot{q}_{2}+K\left(q_{2}-q 1\right)+d t=u
\end{array}\right.
$$

where $q_{i}, \dot{q}_{i}$, and $\ddot{q}_{i}$ are angular displacement, angular velocity, and angular acceleration of the robot $(i=1)$ and motor rotation ( $i=2)$, respectively. $I$ and $J$ are the nominal inertia of the robot and motor, respectively. $K$ is the elastic stiffness of the flexible robot. $M$ and $l$ are the mass and length of the robot joint, respectively. $g$ is gravitational acceleration, and $u$ is the control moment. $|d t| \leq D$ is the total disturbance of the robot system that is composed of external disturbance, friction torque, and unmodeled dynamics.

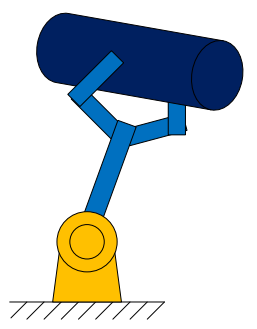

Fig. 1. Structure of the flexible multi-joint robot

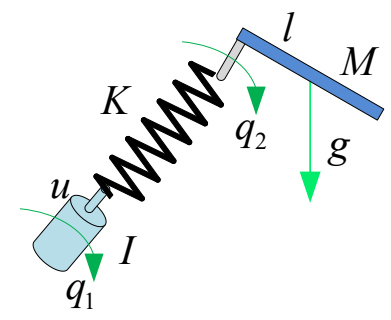

Fig. 2. Spatial model of the flexible multi-joint robot

The new state variable is defined as $x_{1}=q_{1}, x_{2}=\dot{q}_{1}$, $x_{3}=q_{2}$, and $x_{4}=\dot{q}_{2}$. Then, Eq. (1) can be expressed as:

$\left\{\begin{array}{l}\dot{x}_{1}=x_{2} \\ \dot{x}_{2}=-\left[M g l \sin x_{1}+K\left(x_{1}-x_{3}\right)\right] / I \\ \dot{x}_{3}=x_{4} \\ \dot{x}_{4}=\left[u-K\left(x_{3}-x_{1}\right)-d t\right] / J\end{array}\right.$

The control objective represents the flexible joint robot system with uncertain matching in Eq. (2). In this scheme, only the joint angle position can be measured in the system state, whereas the other three state variables are not measurable. The joint position and speed precise tracking of the robot can be realized by designing the control torque $\tau$.

\subsection{Design of flexible joint robot K-observer}

To exclude $x_{3}$ in $\dot{x}_{4}=\left[u-K\left(x_{3}-x_{1}\right)-d t\right] / J$, the system should be controlled only with measurable signals $x_{1}=q_{1}$. Moreover, Eq. (2) needs to be transformed, and $D^{n}$ is expressed as $d^{n} / d t^{n}$. As the system can only measure the joint angle $x_{1}=q_{1}$ of the robot in Eq.(2), the output is set as $y=x_{1}=q_{1}$.

According to Eq. (2), the following equation can be obtained:

$$
D^{2} y=\left(-M g l \sin x_{1}-K x_{1}+K x_{3}\right) / I
$$

Meanwhile, according to Eq. (3), the following equations can be obtained:

$$
\begin{aligned}
& x_{3}=\left(I D^{2} y+M g l \sin y+K y\right) / K \\
& x_{4}=\left(I D^{3} y+M g l D \sin y+K D y\right) / K \\
& \dot{x}_{4}=\left(I D^{4} y+M g l D^{2} \sin y+K D^{2} y\right) / K
\end{aligned}
$$

The dynamic model of the non-interference controlled motor can be expressed as

$$
\dot{x}_{4}=\left[u-K x_{3}-K x_{1}\right] / J
$$

Eqs. (6) and (7) can be expressed as

$$
\begin{aligned}
& \left(I D^{4} y+M g l D^{2} \sin y+K D^{2} y\right) / K= \\
& u / J-\left(I D^{2} y+M g l \sin y\right) / J
\end{aligned}
$$

Then, the following equation can be obtained: 
$D^{4} y+a_{1} D^{2} y+a_{2} D^{2} \sin y+a_{3} \sin y=b_{0} u$

where $\quad a_{1}=K(I+J) /(I J)>0 \quad, \quad a_{2}=M g l / I>0 \quad$, $a_{3}=\mathrm{KMgl} /(I J)>0$, and $b_{0}=K /(I J)>0$. The four parameters are time-varying and bounded, in which $a_{i \text { min }} \leq\left\|a_{i}\right\| \leq a_{i \max }, i=1,2,3$ and $b_{\text {min }} \leq\left\|b_{0}\right\| \leq b_{\text {max }}$.

Let $z_{1}=y, z_{2}=D y$ and $z_{3}=D^{2} y+a_{1} y+a_{2} \sin y$.

Then, the following equations can be obtained:

$$
\left\{\begin{array}{l}
\dot{z}_{1}=z_{2} \\
\dot{z}_{2}=z_{3}-a_{1} z_{1}-a_{2} \sin z_{1} \\
\dot{z}_{3}=z_{4} \\
\dot{z}_{4}=-a_{3} \sin z_{1}+b_{0} u \\
y=z_{1}
\end{array}\right.
$$

Eq. (10) has the same input and output as the undisturbed Eq. (2), in which $z_{1}=x_{1}=q_{1}$ represents the position angle of the robot joint, $z_{2}=D x_{1}$ represents the angular velocity of the robot joint, and $z_{3}$ and $z_{4}$ have no clear physical meaning and are dimensionless.

The state equation of Eq. (10) can be expressed as:

$$
\left\{\begin{array}{l}
\dot{z}=A z+a_{1} f_{1}+a_{2} f_{2}+a_{3} f_{3}+b_{0} u \\
y=c^{T} z
\end{array}\right.
$$

where,

$$
\begin{aligned}
& z=\left[\begin{array}{c}
z_{1} \\
z_{2} \\
z_{3} \\
z_{4}
\end{array}\right], \quad A=\left[\begin{array}{llll}
0 & 1 & 0 & 0 \\
0 & 0 & 1 & 0 \\
0 & 0 & 0 & 1 \\
0 & 0 & 0 & 0
\end{array}\right], \quad b=\left[\begin{array}{l}
0 \\
0 \\
0 \\
b_{0}
\end{array}\right], \quad f_{1}=\left[\begin{array}{c}
0 \\
z_{1} \\
0 \\
0
\end{array}\right], \\
& f_{2}=\left[\begin{array}{c}
0 \\
\sin z_{1} \\
0 \\
0
\end{array}\right], f_{3}=\left[\begin{array}{c}
0 \\
0 \\
0 \\
\sin z_{1}
\end{array}\right], \text { and } z=\left[\begin{array}{l}
1 \\
0 \\
0 \\
0
\end{array}\right] .
\end{aligned}
$$

The K-observer is designed, and the vector is selected and transformed into Hurwitz equations.

$$
\left\{\begin{array}{l}
\dot{\hat{z}}=\omega+a_{1} \varphi_{1}+a_{2} \varphi_{2}+a_{3} \varphi_{3}+b_{0} v \\
\dot{\omega}=A_{0} \omega+k z_{1} \\
\dot{\varphi}_{1}=A_{0} \varphi_{1}+f_{1} \\
\dot{\varphi}_{2}=A_{0} \varphi_{2}+f_{2} \\
\dot{\varphi}_{3}=A_{0} \varphi_{3}+f_{3} \\
\dot{v}=A_{0} v+g_{4} u
\end{array}\right.
$$

where $\omega, \varphi_{1}, \varphi_{2}, \varphi_{3}$, and $v$ are observer state vectors, in which $e_{4}=\left[\begin{array}{llll}0 & 0 & 0 & 1\end{array}\right]^{T}$ and $v=\left[\begin{array}{llll}v_{1} & v_{2} & v_{3} & v_{4}\end{array}\right]^{T}$.

The observer estimation error is defined as $\tilde{z}=z-\hat{z}$. Then, the following equation can be obtained:

$$
\begin{aligned}
\dot{\tilde{z}}= & \dot{z}-\dot{\hat{z}}=A z+a_{1} f_{1}+a_{2} f_{2}+a_{3} f_{3}+b_{0} u- \\
& \left(\omega+a_{1} \varphi_{1}+a_{2} \varphi_{2}+a_{3} \varphi_{3}+b_{0} v\right) \\
= & \left(A_{0}+k c^{T}\right) z+b u-\left(A_{0} \hat{z}+k z_{1}+b_{0} g_{4} u\right) \\
= & A_{0}(z-\hat{z})=A_{0} \tilde{z}
\end{aligned}
$$

As $A_{0}$ is a Hurwitz's formulation, $z$ converges exponentially to zero, i.e., the state observation of the flexible joint robot is realized.

\subsection{Design of the flexible joint robot-adaptive dynamic} surface controller

On the basis of the unknown state of Eq. (10) that is reconstructed by the $K$-observer and in consideration of the existence of the external disturbance $\|d\| \leq \Delta$, Eq. (13) is used as the design of the position tracking controller to realize the position tracking of the flexible joint robot, i.e., to achieve through the objectives $x_{1} \rightarrow x_{d}$ and $\dot{x}_{1} \rightarrow \dot{x}_{d}$ the control target and avoid the differential explosion phenomenon in the design.

$\left\{\begin{array}{l}\dot{z}_{1}=z_{2} \\ \dot{z}_{2}=z_{3}-a_{1} z_{1}-a_{2} \sin z_{1} \\ \dot{z}_{3}=z_{4} \\ \dot{z}_{4}=-a_{3} \sin z_{1}+b_{0} u+d \\ y=z_{1}\end{array}\right.$

The first error surface is defined as:

$\left\{\begin{array}{l}\dot{\hat{z}}=\omega+a_{1} \varphi_{1}+a_{2} \varphi_{2}+a_{3} \varphi_{3}+b_{0} v \\ \dot{\omega}=A_{0} \omega+k z_{1} \\ \dot{\varphi}_{1}=A_{0} \varphi_{1}+f_{1} \\ \dot{\varphi}_{2}=A_{0} \varphi_{2}+f_{2} \\ \dot{\varphi}_{3}=A_{0} \varphi_{3}+f_{3} \\ \dot{v}=A_{0} v+g_{4} u\end{array}\right.$

Then,

$$
\begin{aligned}
\dot{s}_{1} & =z_{2}-\dot{z}_{1 d}=\omega_{2}+a_{1} \varphi_{12}+a_{2} \varphi_{22}+a_{3} \varphi_{32}+b_{0} v_{2}+\tilde{z}_{2}-\dot{z}_{1 d} \\
& =b_{0}\left[v_{2}+\frac{a_{1}}{b_{0}} \varphi_{12}+\frac{a_{2}}{b_{0}} \varphi_{22}+\frac{a_{3}}{b_{0}} \varphi_{32}+\frac{1}{b_{0}}\left(\omega_{2}-\dot{z}_{1 d}+\tilde{z}_{2}\right)\right]
\end{aligned}
$$

The vector is defined as:

$\left\{\begin{array}{l}\psi=\left[\begin{array}{llll}\varphi_{12} & \varphi_{22} & \varphi_{32} & l_{1} s_{1}+\omega_{2}-\dot{z}_{1 d}\end{array}\right] \\ \theta=\left[\begin{array}{llll}\theta_{1} & \theta_{2} & \theta_{3} & \theta_{4}\end{array}\right]^{T}\end{array}\right.$

where $l_{1}>0$ is the design parameter, and $\left[\begin{array}{llll}\theta_{1} & \theta_{2} & \theta_{3} & \theta_{4}\end{array}\right]^{T}=\left[\begin{array}{llll}a_{1} / b_{0} & a_{2} / b_{0} & a_{3} / b_{0} & 1 / b_{0}\end{array}\right]^{T}$ such that $\dot{s}_{1}=b_{0} v_{2}+\theta^{T} \psi$.

The designed virtual control is given by

$\bar{v}_{2}=-\hat{\theta}^{T} \psi$ 
where $\hat{\theta}=\left[\begin{array}{llll}\hat{\theta}_{1} & \hat{\theta}_{2} & \hat{\theta}_{3} & \hat{\theta}_{4}\end{array}\right]^{T}$ is an estimate of $\theta$, and it satisfies

$$
\dot{\hat{\theta}}=\Lambda \psi s_{1}-\Lambda \eta \hat{\theta}
$$

where $\Lambda=\Lambda^{T}>0$ is a set of the symmetric matrix, and $\eta>0$ corresponds to the design parameters.

A low-pass filter with a filter time constant $\tau_{2}$ is designed such that the system can obtain a new state variable $v_{2 d}$.

$\tau_{2} \dot{v}_{2 d}+v_{2 d}=\bar{v}_{2}, v_{2 d}(0)=\bar{v}_{2}(0)$

The second error surface is defined as

$s_{2}=v_{2}-v_{2 d}$

Then,

$\dot{s}_{2}=-k_{2} v_{1}+v_{3}-\dot{v}_{2 d}$

The designed virtual control is given by

$\bar{v}_{3}=-l_{2} s_{2}+k_{2} v_{1}+\dot{v}_{2 d}$

where $l_{2}>0$ corresponds to the design parameters.

A low-pass filter with a filter time constant $\tau_{3}$ is designed to obtain a new state variable $v_{3 d}$.

$\tau_{3} \dot{v}_{3 d}+v_{3 d}=\bar{v}_{3}, v_{3 d}(0)=\bar{v}_{3}(0)$

The third error surface is defined as

$s_{3}=v_{3}-v_{3 d}$

Then,

$\dot{s}_{3}=-k_{3} v_{1}+v_{4}-\dot{v}_{3 d}$

The designed virtual control is given by

$\bar{v}_{4}=-l_{3} s_{3}+k_{3} v_{1}+\dot{v}_{3 d}$

where $l_{3}>0$ corresponds to the design parameters.

A low-pass filter with filter time constant $\tau_{4}$ is designed to obtain a new state variable $v_{4 d}$.

$\tau_{4} \dot{v}_{4 d}+v_{4 d}=\bar{v}_{4}, v_{4 d}(0)=\bar{v}_{4}(0)$

The fourth error surface is defined as

$S_{4}=v_{4}-v_{4 d}$

Then,

$\dot{s}_{4}=-k_{4} v_{1}+u-\dot{v}_{4 d}+d$
The designed actual control is given by

$u=-l_{4} s_{4}+k_{4} v_{1}+\dot{v}_{4 d}-\Delta$

where $l_{4}>0$ is the design parameter.

As depicted by the process shown above, the design only uses the state, and the adaptive law only realizes the estimation of all unknown parameters in the first step of the design. This approach simplifies the design process and reduces the amount of calculation.

The virtual control error is defined as

$\varepsilon_{i}=v_{i d}-\bar{v}_{i},(i=2,3,4)$

The parameter estimation error is defined as

$\tilde{\theta}=\hat{\theta}-\theta$

The Lyapunov function is given by

$V=V_{1}+V_{2}+V_{3}$,

where $\quad V_{1}=\frac{1}{2} \sum_{i=1}^{4} s_{i}^{2} \quad, \quad V_{2}=\frac{1}{2} \sum_{i=2}^{4} y_{i}^{2} \quad, \quad$ and $V_{1}=\frac{1}{2} b_{0} \tilde{\theta}^{T} \Lambda^{-1} \tilde{\theta}+\tilde{z}^{T} P \tilde{z}$. $P$ represents a symmetric positive definite matrix.

Theorem. For the non-linear system with uncertain parameters and external disturbances after the transformation, Eq. (13) adopts the observer shown in Eq. (12), the adaptive rate shown in Eq. (16), and the control rate shown in Eq. (30). The controller structure is shown in Fig. 3 . If the controlled system in Eq. (13) satisfies a specific set of requirements (i.e., (1) only the joint position of the flexible robot $x_{1}=q_{1}$ can be measured, and other states are not measurable; (2) the upper and lower bounds of the uncertain parameters $a_{i}(i=1,2,3,4)$ are known; (3) the expected trajectory $z_{1 d}$ is bounded and has its first and second derivatives, and the positive number $\varsigma$ satisfies $z_{1 d}^{2}+\dot{z}_{1 d}^{2}+\ddot{z}_{1 d}^{2} \leq \varsigma^{2}$; and (4) the initial condition of the Lyapunov function is $V(0)<\chi$, where $\chi$ is any constant), then the adjusting parameters are given by $c_{i}(i=1,2,3,4)$, $\tau_{i}(i=1,2,3,4), \eta$, and $\Lambda$. Thus, the tracking error can converge to any small residual set.

It has been proven that the derivation of $V_{1}, V_{2}$, and $V_{3}$ can be obtained as follows:

$$
\begin{aligned}
& \dot{V}_{1}=S_{1}\left(b_{0} S_{2}+b_{0} y_{2}-b_{0} \tilde{\theta}^{T} \Psi-l_{1} S_{1}+\tilde{z}_{2}\right)+ \\
& \quad \mathrm{S}_{2}\left(S_{3}+y_{3}-l_{2} S_{2}\right)+S_{3}\left(S_{2}+y_{3}-l_{3} S_{3}\right) l_{4} S_{2}^{2} \\
& \dot{V}_{2} \leq \sum_{2}^{4}\left(-y_{i}^{2} / \tau_{i}+B_{i}\left|y_{i}\right|\right)_{3}
\end{aligned}
$$

$\dot{V}_{3}=b_{0} \tilde{\theta}^{T} S_{1} \Psi-b_{0} \tilde{\theta}^{T} \eta \hat{\theta}-\tilde{z}^{T} \tilde{z}$

After using Eqs. (34)-(36), Young's inequality is also adopted for the simplification. Eq. (37) can be obtained as 


$$
\begin{aligned}
\dot{V}= & \left(b_{0}+\frac{1}{2}-l_{1}\right) S_{1}^{2}+\left(b_{0}+\frac{1}{2}-l_{2}\right) S_{2}^{2}+ \\
& \left(\frac{3}{2}-l_{3}\right) S_{3}^{2}+\left(\frac{1}{2}-l_{4}\right) S_{4}^{2}+ \\
& +\left(\frac{B_{2}^{2}}{2}+\frac{b_{0}}{2}-\frac{1}{\tau_{2}}\right) \mathrm{y}_{2}^{2}+\left(\frac{B_{3}^{2}}{2}+\frac{1}{2}-\frac{1}{\tau_{3}}\right) \mathrm{y}_{3}^{2}+ \\
& +\left(\frac{B_{4}^{2}}{2}+\frac{1}{2}-\frac{1}{\tau_{4}}\right) \mathrm{y}_{4}^{2}-\eta b_{0} \tilde{\theta}^{T} \Gamma^{-1} \theta+ \\
& \frac{3}{2}+\frac{b_{0} \eta}{2}\|\theta\|^{2}+E(\tilde{z})
\end{aligned}
$$

where $l_{1} \geq b_{M}+\frac{1}{2}+\kappa, l_{2} \geq \frac{b_{M}}{2}+\frac{1}{2}+\kappa, l_{3} \geq \frac{3}{2}+\kappa, l_{4} \geq \frac{1}{2}+\kappa$, $\frac{1}{\tau_{2}} \geq M_{2}^{2} / 2+\frac{b_{M}}{2}+\kappa, \frac{1}{\tau_{3}} \geq M_{3}^{2} / 2+\frac{1}{2}+\kappa, \frac{1}{\tau_{4}} \geq M_{4}^{2} / 2+\frac{1}{2}+\kappa$, $\eta \geq 2 \kappa \lambda_{\max }\left(\Gamma^{-1}\right)$, and $E(\tilde{z})=\frac{1}{2} \tilde{z}_{2}^{2}-\tilde{z}^{T} \tilde{z}$. In these formulas, $\kappa>0$ is the designed parameter, $l_{4} \geq \frac{1}{2}+\kappa, b_{M}>0$ is the maximum value of $b_{0}$, and $\lambda(*)$ is the maximum eigenvalue of the matrix *.

$$
\text { If } Q=\frac{3}{2}+b_{0} \eta\|\theta\|^{2} / 2 \text {, then }
$$

$$
\dot{V} \leq-2 \kappa V+Q+\varepsilon
$$

Considering that $V(0)<\chi$, the integral of two sides can be obtained as

$$
V \leq \frac{1}{2} Q / \kappa+\left[V(0)-\frac{1}{2}(Q+\varepsilon) / \kappa\right] e^{-2 \kappa t}
$$

Therefore, all signals of the closed-loop controlled system are semi-globally and uniformly bounded in Eq. (13). Moreover, Eq. (13) satisfies $\lim _{t \rightarrow 0} V \leq Q / \kappa$. By adjusting the design parameters $r$, the tracking error of the system can converge to any small value.

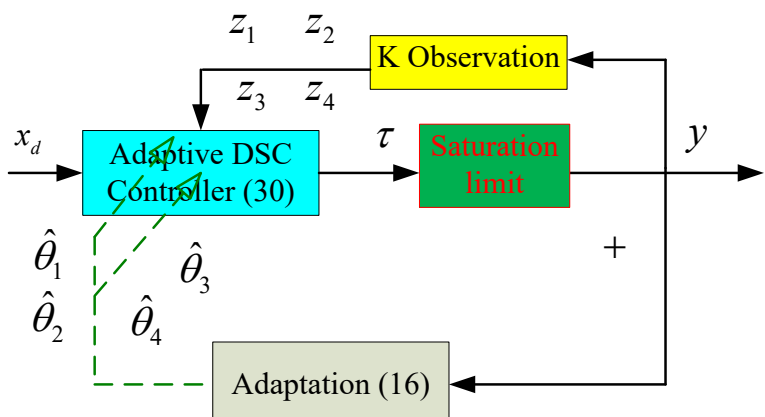

Fig. 3. Structure diagram of the adaptive dynamic surface controller based on the K observer

\section{Result analysis and discussion}

On the basis of the dynamic model of the flexible joint robot in Eq. (2), the nominal parameters of the unknown physical quantities are taken as $I=1 \mathrm{~kg} \cdot \mathrm{m}^{2}, \quad J=1 \mathrm{~kg} \cdot \mathrm{m}^{2}$, $K=40 \mathrm{~N} \cdot \mathrm{m} / \mathrm{rad}$, and $\mathrm{Mgl}=5 \mathrm{~N} \cdot \mathrm{m}$. The initial state is $\left[\begin{array}{llll}q_{1} & \dot{q}_{1} & q_{2} & \dot{q}_{2}\end{array}\right]=\left[\begin{array}{llll}0.5 & 0 & 0 & 0\end{array}\right]$. The performance of the designed observer in Eq. (12) and the controller in Eq. (30) are verified by simulation.

\subsection{Observer performance}

The system input is $u=\sin t$. The observation of the system state and the observation error are shown in Figs. 4 and 5, respectively.

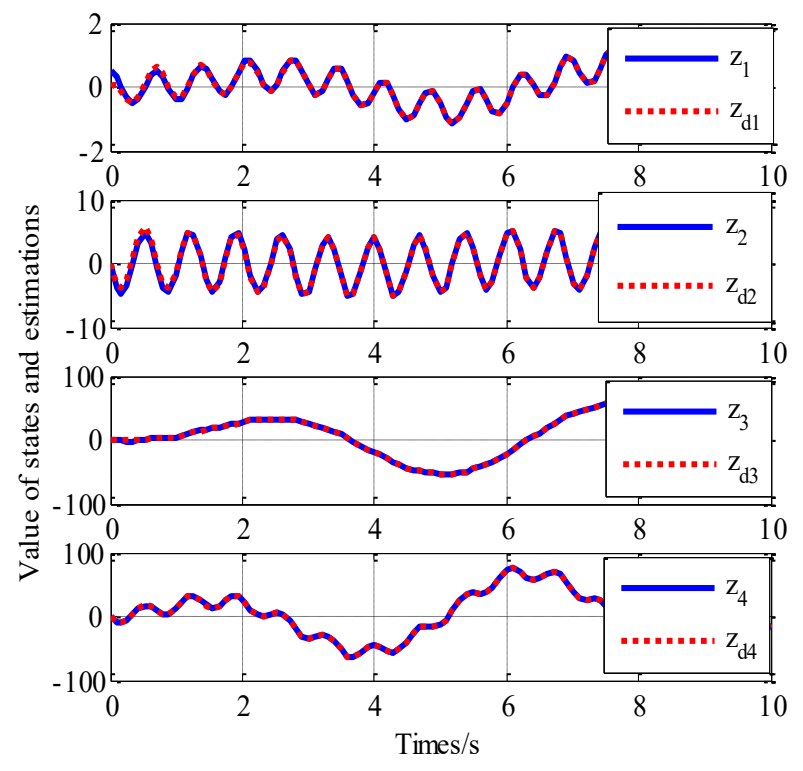

Fig. 4. K-state observation curve of the flexible robot

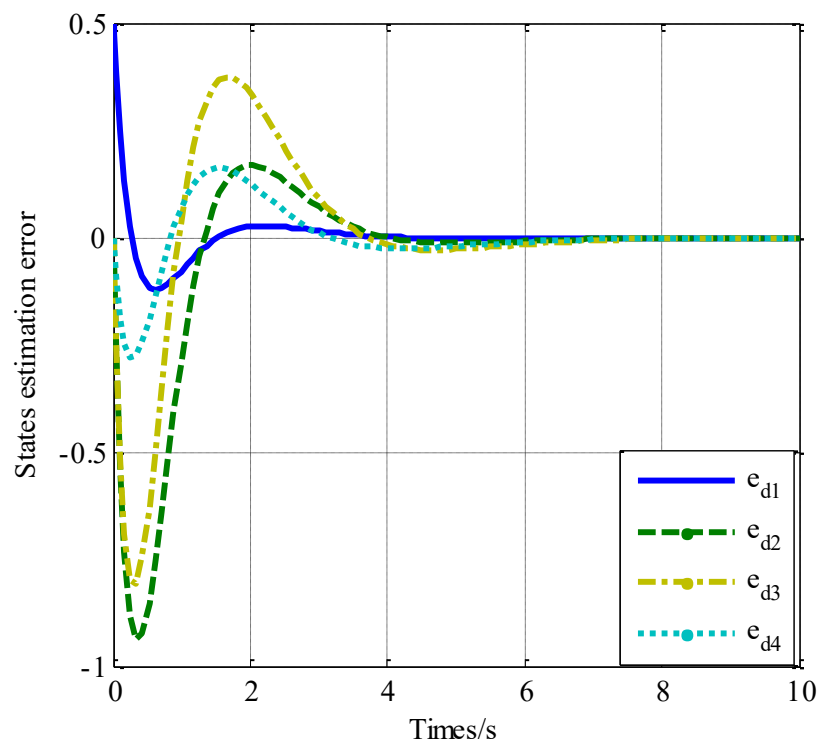

Fig. 5. $K$-observer error curve of the flexible robot

Figs. 4 and 5 show that when only the joint angle position of the flexible robot is known in the system, its state tracking can be realized using the $K$-state observer at 4 s. The tracking error of the state $z_{2}$ and $z_{3}$ are large and within a one-unit dimension.

\subsection{Controller performance}

The desired trajectory is set as $z_{1 d}=x_{d}=\sin t$, and the controller parameters are set as $\left[\begin{array}{llll}k_{1} & k_{2} & k_{3} & k_{4}\end{array}\right]=\left[\begin{array}{llll}10 & 50 & 110 & 80\end{array}\right] \quad$, $\left[\begin{array}{llll}l_{1} & l_{2} & l_{3} & l_{4}\end{array}\right]=\left[\begin{array}{llll}10 & 9 & 6 & 4\end{array}\right]$ $\Gamma=\operatorname{diag}\left\{\begin{array}{llll}950 & 60 & 830 & 0.3\end{array}\right\}$, and $\left.\tau_{i}\right|_{i=2,3,4}=0.2$. Fig. 6 shows the curves of the joint angle tracking, error response, and control input of the inverse control flexible robot without a model uncertainty. Meanwhile, Fig. 7 shows the curves of the joint angle tracking, error response, and control input of the dynamic surface control flexible robot without a model uncertainty. 

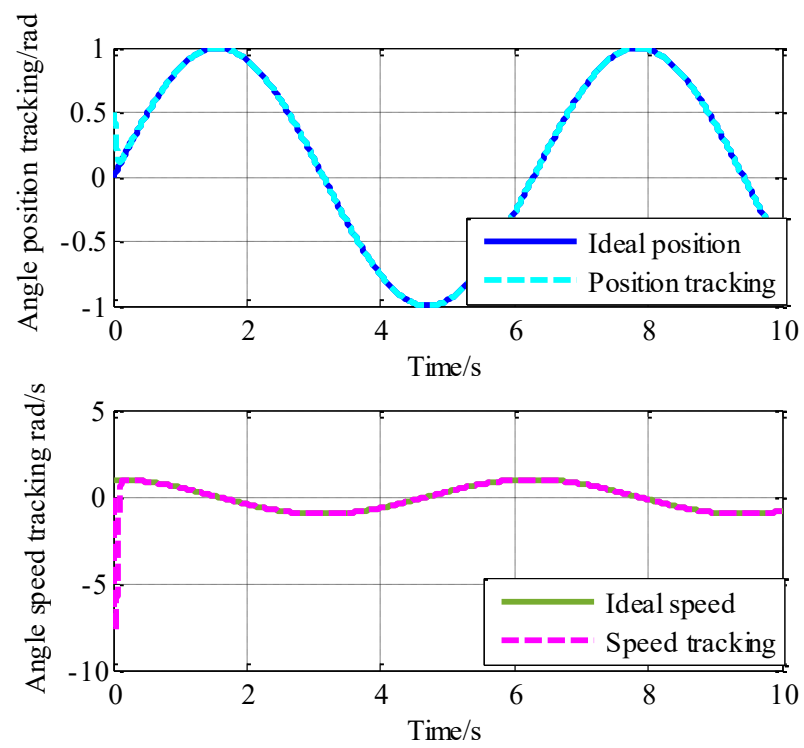

(a) Joint angle position tracking curve and error curve of the flexible robot

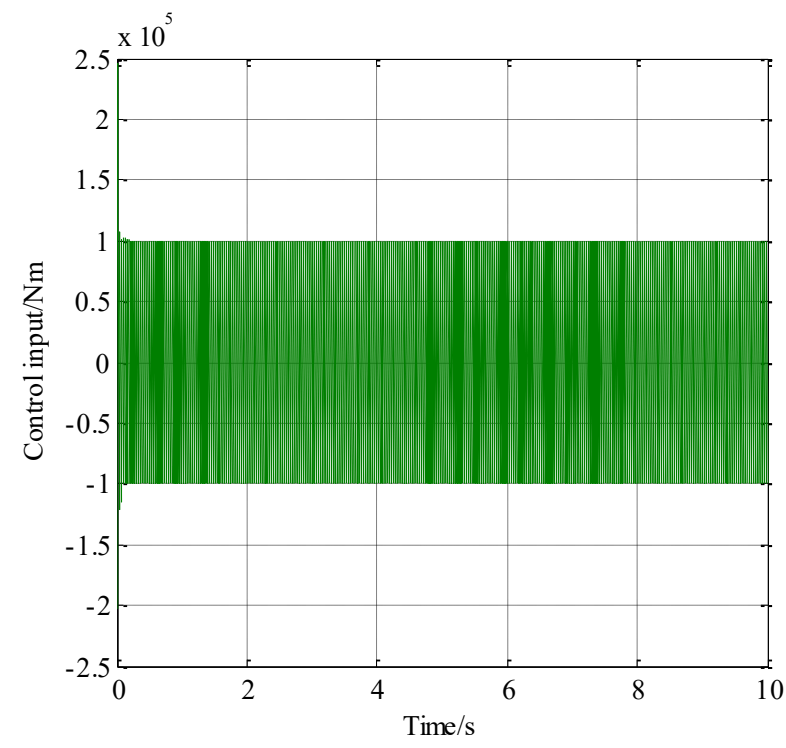

(b) Input curve of the flexible robot control

Fig. 6. Tracking and control input curves of the flexible robot inverse control without any uncertainty

As shown in Fig. 6, when no uncertain parameters exist in the system, the backstepping control can realize the angular position and angular velocity tracking of the robot joint at $0.12 \mathrm{~s}$. The angular position error gradually decreases from $0.5 \mathrm{rad}$ to zero and the angular velocity error is large, reaching $7 \mathrm{rad} / \mathrm{s}$. The input of the backstepping control has a large chattering due to the expansion of the system differential term in the derivative process of the virtual control, indicating a highly complex controller.

As shown in Fig. 7, when no uncertain parameters exist in the system, the dynamic surface control has a better tracking performance than the backstepping control. The angle position error of the dynamic surface control decreases rapidly from $0.5 \mathrm{rad}$ to zero and the maximum error of angular velocity are within $3 \mathrm{rad} / \mathrm{s}$, which is 1.3 times higher than that of the inversion control. The dynamic surface method has a large control input fluctuation in the actual stage; once stable, the control input torque is within $180 \mathrm{~nm}$, which considerably reduces the fluctuation.
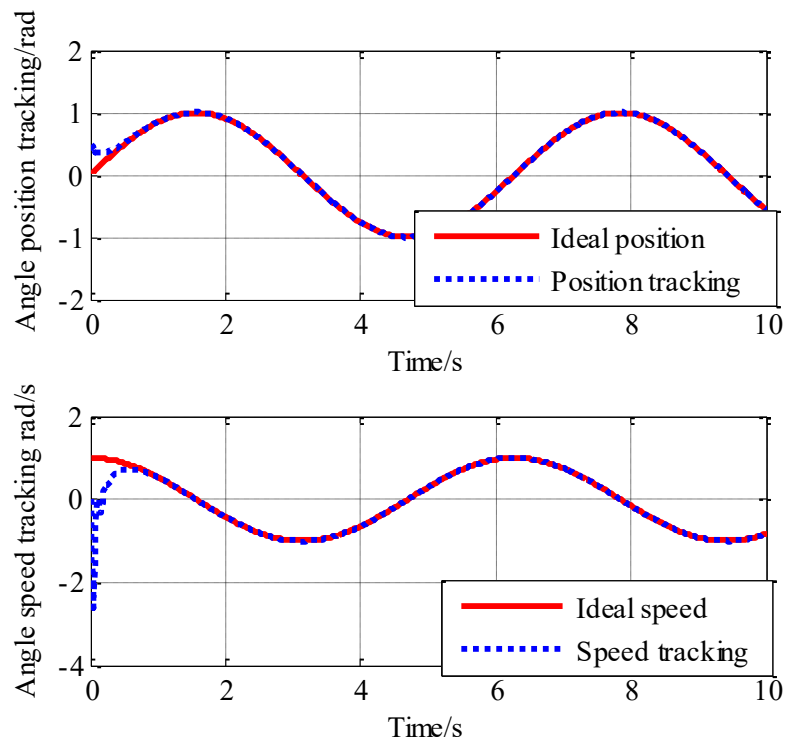

(a) Joint angular position and angular velocity tracking curve of the flexible robot

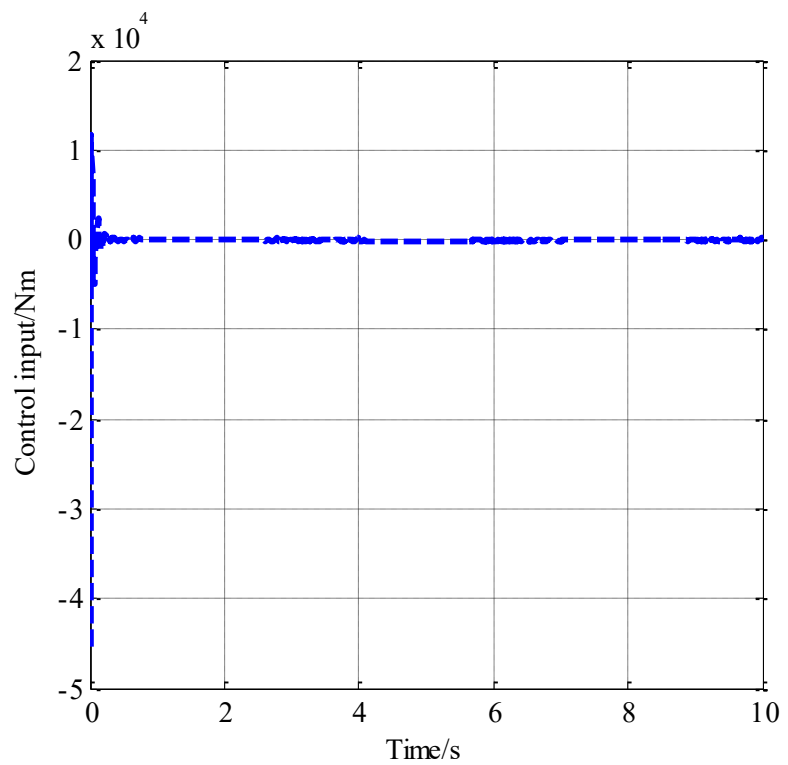

(b) Control input curve of the flexible robot

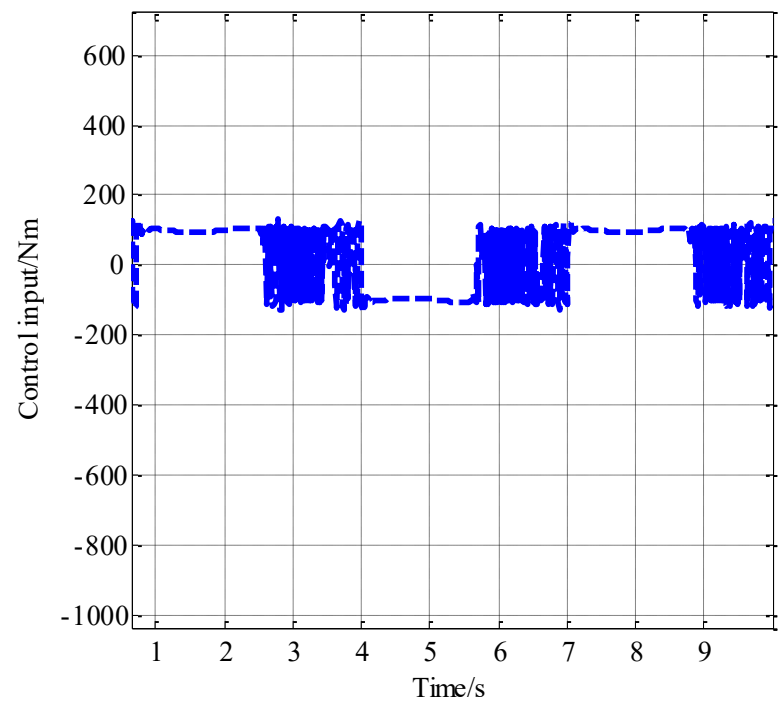

(c) Steady state amplification curve of the flexible robot control input Fig. 7. Tracking and control input curves of the flexible robot dynamic surface control without uncertainty 
The control described above is performed under the conditions of system parameter determination and state observability. When the uncertain parameters and state are not completely measurable, the abovementioned method is powerless. The adaptive dynamic surface control based on the proposed $K$-observer is subsequently implemented with these two factors considered at the same time. The simulation results are shown in Figs. 8 and 9.
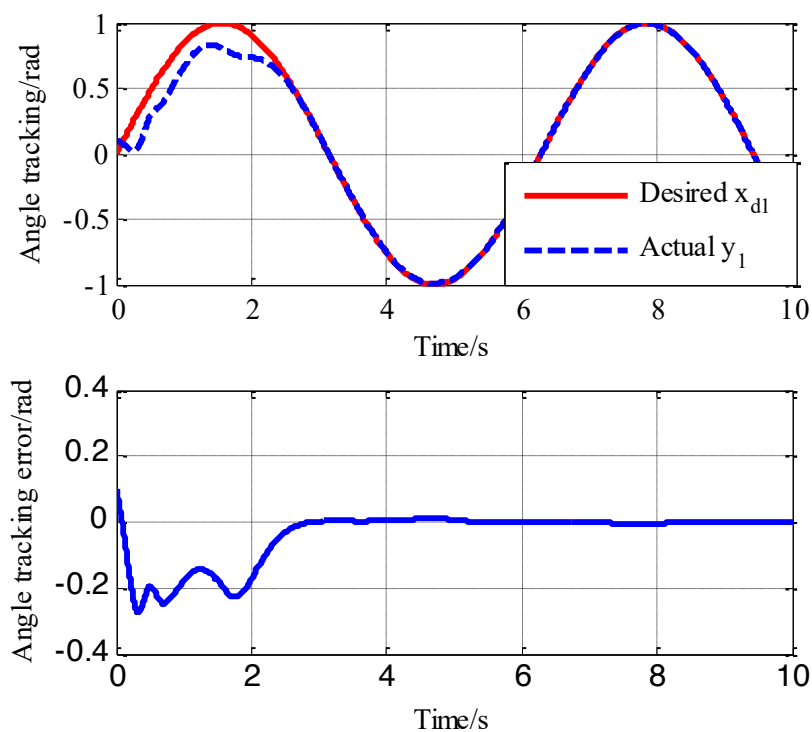

(a) Joint angle position tracking curve and error curve of the flexible robot

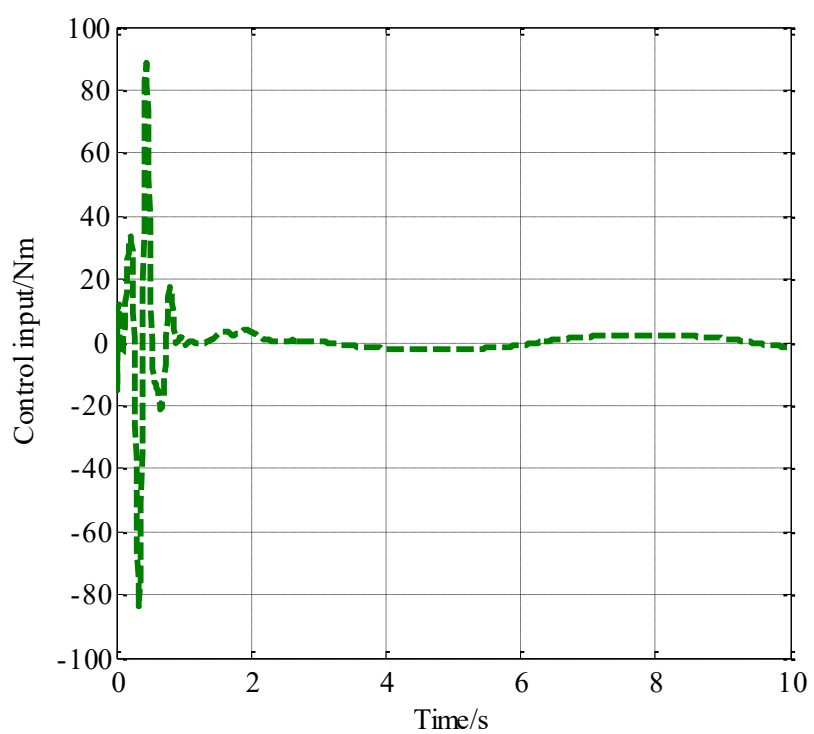

(b) Control input curve of the flexible robot

Fig. 8. Tracking and control input curves of the flexible robot with uncertainties $\backslash$

As shown in Figs. 8 and 9, when parameter uncertainties and incompletely measurable state both exist in the system, the following conclusions can be drawn: (1) The dynamic tracking time of the system increases considerably, while the speed of the tracking error approaching zero slows down. The control performance of the system is affected by the dynamic estimation performance of the uncertain parameters, as shown in Fig. 9. The parameter estimation is realized at $2.5 \mathrm{~s}$, and the robot joint can track the angular position at this time. (2) The control input curve of this method has a small fluctuation at the initial stage and a small control torque in the steady-state phase. (3) The $K$-state observer can estimate the unknown parameters at $2.5 \mathrm{~s}$, indicating high estimation accuracy.
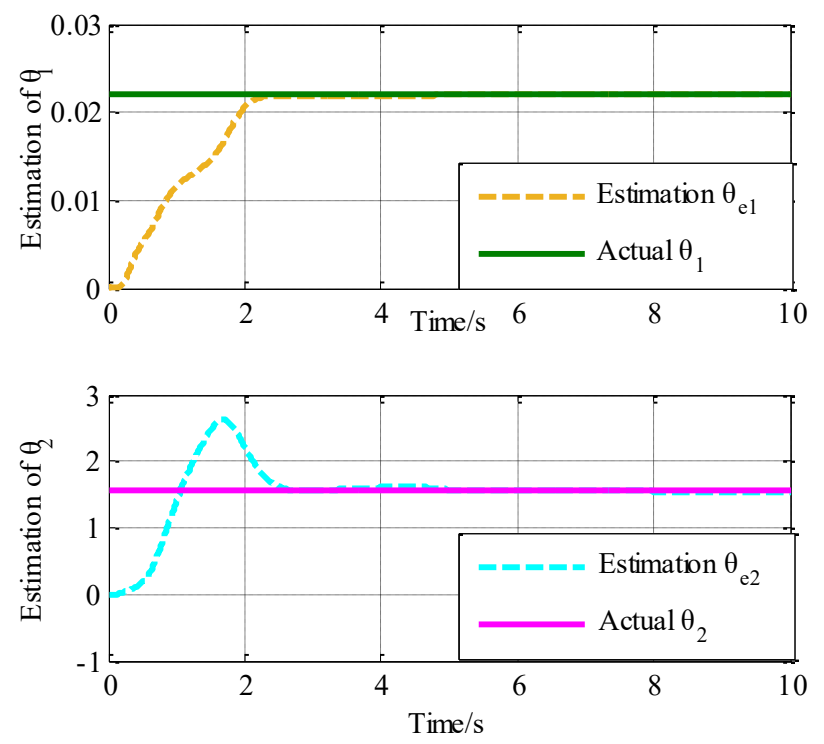

(a) Estimation curve of parameters $\theta_{1}$ and $\theta_{2}$
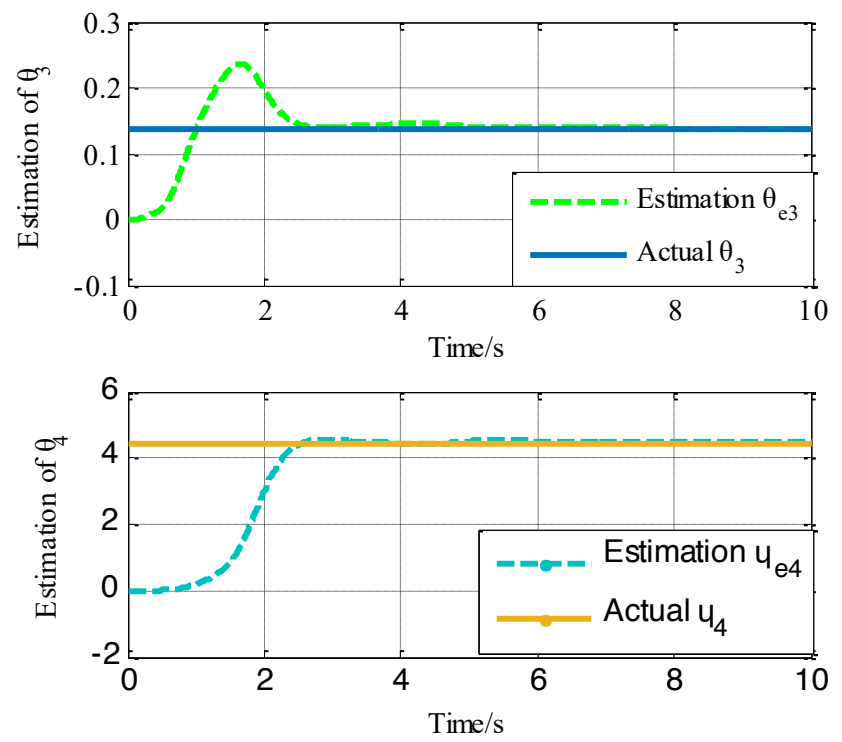

(b) Estimation curve of parameters $\theta_{3}$ and $\theta_{4}$

Fig. 9. Estimation curve of the flexible robot with uncertain parameters

\section{Conclusion}

In view of overcoming the influences of parameter perturbation and incompletely measurable state on the joint tracking accuracy of a flexible robot system, the system performance based on the characteristics of the flexible robot was analyzed by reconstructing system state equation and by combining state observation theory, adaptive control theory, and the dynamic surface design method. The following conclusions could be drawn:

(1) The designed $K$-state observer can realize the realtime observation of the unknown state in the system.

(2) By combining adaptive theory and the dynamic surface design method, the uncertain parameters can be estimated quickly within $2.5 \mathrm{~s}$, and the differential explosion caused by the back-stepping control is effectively avoided. 
(3) The control output of the proposed method is smoother than the back-stepping control and conventional dynamic surface control, and its control energy is smaller.

(4) The proposed method can effectively reduce the constraints of the inversion control and conventional dynamic surface control on a fully measurable state of the system.

By combining simulation experiment and theory, this study has proposed an effective approach of restraining the influence of uncertain parameters of the flexible robot system model and the fully measurable state limit. The control method has a simple structure, is clear and easy to understand, and is highly suitable for actual working conditions. The proposed method has certain guiding significance for the subsequent application of flexible robots in the field of high-precision assembly.

\section{Acknowledgements}

This study was supported by the Key Scientific Study Projects of Higher Education Institutions of Henan Province (Grant Nos. 20B470003 and 18B470007) and the Promotion Special Project of the Scientific Study Program of Henan Province (Grant Nos. 202102210084 and 202102210298).

This is an Open Access article distributed under the terms of the Creative Commons Attribution License

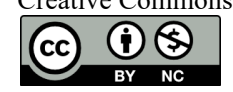

\section{References}

1. Zhou Y. P., Lou J. Q., Chen T. H., Ma J. Q., Wei Y. D., "Coupling dynamic modeling of a flexible manipulator driven by servo joint and test recognition". Journal of Vibration and Shock, 38(9), 2019, pp.277-284.

2. Lei R. H., Chen L., "Adaptive neural network fault-tolerant control and residual vibration suppression for flexible-arm space robot with attitude-controlled base". Journal of Vibration and Shock, 39(7), 2020, pp.156-162.

3. Du X. L., Du J. L., Bao H., Zhong W., Cui K., "Dynamic analysis of deployable antennas considering truss flexibility". Journal of Mechanical Engineering, 56(7), 2020, pp.119-126.

4. Guo Y. N., Wang P. Y., Jin S., "A new optimization approach to the flexible spacecraft actuators placement". Flight Control and Detection, 2(5), 2019, pp.49-54.

5. Duan H. F, Han W., Jiang L. Z., Chen Y. S., "Design of flexible assembly automation system for socket switch based on industrial robot". Manufacturing Technology and Machine Tool, (3), 2020, pp.13-16.

6. Meng F. G., Wang W., Li X. P., Liu Y. P., "A robot off-line programming system for typical space line cutting". Machinery Design and Manufacture, (9), 2019, pp.236-239.

7. Tu Q., Yue D. H., Wang Y.J., Liu G. X., "Design and investigation on the deformation characteristics of soft robot hands for lateral grasping of fruit and vegetables". Machine Tool and Hydraulics, 47(9), 2019, pp.51-57.

8. Feng Y. B., Zhao X. H., He Z., Li Z. G, Wang Y. B., "Depth hover control of spherical robot for internal detection of oil-immersed transformer". Control and Decision, 35(2), 2020, pp.375-381.

9. Huang X. R., Song Y. Y., Li Q. S., Xiao X., "A vibration suppression algorithm for industrial robot joint servo system based on internal model control". Transactions of China Electrotechnical Society, 34(3), 2019, pp.497-505.

10. Liu L. Y., "Design and application of internal model control for jointed cloud robot with delay time". Journal of Shijiazhuang University of Applied Technology, 31(4), 2019, pp.8-12.

11. Fu B. C., Wang H., Qiu W. Q., Xue B., "Flexible joint manipulators control strategy based on PID control". Journal of Anhui Polytechnic University, 31(2), 2016, pp.45-48.

12. Sarkhel P., Banerjee N., "Hui N.B. Fuzzy logic-based tuning of PID controller to control flexible manipulators". Springer International Publishing, 2(4), 2020, pp.367-377.

13. Pradhan S. K., Subudhi B., "Position control of a flexible manipulator using a new nonlinear self-tuning PID controller". IEEE/CAA Journal of Automatica Sinica, 7(1), 2020, pp.136-149.

14. Malzahn J., Bertram T., "On the equivalence of direct strain feedback and lumped parameter wave echo control for oscillation damping of elastic-link arms". IEEE Robotics and Automation Letters, 1(1), 2016, pp.447-454.
15. Endo T., Umemoto K., Matsuno F., "Exponential stability of dual flexible arms for grasping and orientation control". The Institution of Engineering and Technology Control Theory and Applications, 13(16), 2019, pp.2546-2555.

16. Endo T., Umemoto K., Matsuno F., Jia Y., "Contact-force control of a flexible Timoshenko arm in rigid/soft environment". IEEE Transactions on Automatic Control, 62(5), 2017, pp.2546-2553.

17. Luc L. T., Alin A. S., "Robust adaptive tracking control based on state feedback controller with integrator terms for elastic joint robots with uncertain parameters". IEEE Transactions on Control Systems Technology, 26(6), 2018, pp. 2259-2267.

18. Palleschi A., Mengacci R., Angelini F., Caporale D., Pallottino L., Luca A. D., Garabini M., "Time-optimal trajectory planning for flexible joint robots". IEEE Robotics and Automation Letters, 5(2), 2020, pp.938-945.

19. Zhang M., Fang L. J., Sun F., Sun X. W., "Design and decoupling control of variable-stiffness flexible robot joint". Electric Machines and Control, 23(7), 2019, pp.120-128.

20. Li Z. H., Wu C. J., Jiang D., Fan Z. H., Ni J., "The dynamics solving method of flexible joint robot based on equalized state system". Journal of Mechanical Engineering, 56(3), 2020, pp.121-129.

21. Zhu A., Chen L., "Full - order sliding mode control for flexible joint space robot including motor dynamic" Journal of Fuzhou University (Natural Science Edition), 47(6), 2019, pp.779-786.

22. Wang Y., Zhang H. B., Chen Y, F., Ji W., "Terminal sliding mode control of flexible end grinding force of wind turbine blade grinding robot". Computer Integrated Manufacturing Systems, 25(7), 2019, pp.1757-1766.

23. Xue Y., Yu H. S., Wu H. R., "Fuzzy backstepping position control for flexible joint robots". Journal of Qingdao University (Engineering and Technology Edition), 34(2), 2019, pp.56-62.

24. Liu H. S., Jin Y. L., Cheng X., Wang Z. Y., Qi J., Liu Y., "Trajectory tracking control for flexible-joint robot manipulators with bounded torque inputs". Control Theory and Applications, 36(6), 2019, pp.983-992.

25. Ouyang Y., He W., Li X., "Reinforcement learning control of a single-link flexible robotic manipulator". The Institution of Engineering and Technology Control Theory and Applications, 11(9), 2017, pp.1426-1433.

26. Brahmi B., Saad M., Ochoa-Luna C., Rahman M. H., Brahmi A., "Adaptive tracking control of an exoskeleton robot with uncertain dynamics based on estimated time-delay control". IEEE/ASME Transactions on Mechatronics, 2(2), 2018, pp.575-585. 